\title{
Análise da qualidade do queijo coalho vendidos em uma feira livre do município de Campina Grande-PB
}

\author{
Analysis of the quality of coalho cheese sold at a free fair in the municipality of Campina Grande-PB \\ Análisis de la calidad del queso coalho vendido en una feria gratuita en la ciudad de Campina \\ Grande-PB
}

Recebido: 24/05/2021 | Revisado: 30/05/2021 | Aceito: 07/06/2021 | Publicado: 21/06/2021

\author{
Wagner Gomes de Melo \\ ORCID: https://orcid.org/0000-0001-5198-0457 \\ Centro Universitário UniFacisa, Brasil \\ E-mail: wagnerlegionario@hotmail.com \\ Ana Clara Bezerra Felinto \\ ORCID: https://orcid.org/0000-0002-5611-6948 \\ Centro Universitário UniFacisa, Brasil \\ E-mail: anaclarabfelinto@gmail.com \\ Steffany Albuquerque do Bú \\ ORCID: https://orcid.org/0000-0002-4804-4657 \\ Centro Universitário UniFacisa, Brasil \\ E-mail: steffanyalbuquerque12@gmail.com \\ Eduarda Josefa Alves Marçal \\ ORCID: https://orcid.org/0000-0003-0021-9144 \\ Centro Universitário UniFacisa, Brasil \\ E-mail: eduardamarca1826@gmail.com \\ Igor Macêdo de Oliveira \\ ORCID: https://orcid.org/0000-0003-3717-7291 \\ Centro Universitário UniFacisa, Brasil \\ E-mail: Igormacedo1955@gmail.com \\ Joyce Almeida Lima \\ ORCID: https://orcid.org/0000-0002-5113-1903 \\ Centro Universitário UniFacisa, Brasil \\ E-mail: joycealmeida95@outlook.com \\ Juliana Barbosa de Sousa \\ ORCID: https://orcid.org/0000-0002-2228-8004 \\ Centro Universitário UniFacisa, Brasil \\ E-mail: juliana.bs628@gmail.com \\ Mayra da Silva Cavalcanti \\ ORCID: https://orcid.org/0000-0003-1269-5324 \\ Centro Universitário UniFacisa, Brasil \\ E-mail: mayra_cavalcanti@yahoo.com.br
}

\begin{abstract}
Resumo
Produto típico do nordeste brasileiro, o queijo tipo coalho tem uma grande importância econômica e cultural. No estado da Paraíba representa um significativo meio de geração de renda aos produtores de leite, em sua grande maioria produzindo queijos tipo coalho de forma artesanal. Para tanto, é utilizado o leite cru como principal matéria-prima para fabricação dessa iguaria, tão utilizada na gastronomia nordestina. A falta da utilização de métodos de pasteurização da matriz alimentar torna-se um meio propício para a veiculação de contaminação do produto. A ausência das Boas Práticas de Fabricação, associado ao transporte, acondicionamento e comercialização inadequada, deixa o consumidor expostos ao risco de doenças transmitidas por alimentos. Pela importância econômica e cultural no comércio deste produto na cidade avaliada, o presente estudo teve o intuito de analisar a qualidade microbiológica do queijo tipo coalho artesanal comercializados na feira. Assim, foram analisadas 10 amostras de diferentes pontos comerciais da feira de queijo da cidade de Campina Grande, Paraíba. As análises microbiológicas seguiram o protocolo da legislação vigente para tal produto, onde foram avaliados quanto a presença de coliformes totais, Staphylococcus aureus, Salmonela spp. e Listeria monocytogenes. $90 \%$ das amostras analisadas estavam acima do permitido pela normativa para coliformes totais, Salmonela spp. e Listeria monocytogenes. A partir dos resultados das análises microbiológicas, foram demonstradas inadequações dos padrões microbiológicas do queijo tipo coalho comercializados na feira central, por apresentar uma grande quantidade de microrganismos patogênicos acima do limite exigido pela legislação brasileira o que levanta um alerta de saúde pública.
\end{abstract}

Palavras-chave: Produtos Lácteos; Boas práticas de manipulação; Controle de qualidade. 


\begin{abstract}
Typical product of the Brazilian northeast, the coalho type cheese has a great economic and cultural importance for this region. In the state of Paraíba, it represents a significant means of income generation for milk producers, most of them producing cheeses like curds in an artisanal way. Therefore, raw milk is used as the main raw material for the manufacture of this delicacy, so used in northeastern gastronomy. The lack of use of pasteurization methods of the food matrix becomes a favorable means for the dissemination of product contamination. the absence of good manufacturing practices, associated with inadequate transportation, packaging and marketing, leaves consumers exposed to the risk of foodborne illnesses. due to the economic and cultural importance in the trade of this product in the evaluated city, this study aimed to analyze the microbiological quality of the artisanal rennet cheese sold at the fair. Thus, 10 samples from different commercial points of the cheese fair in the city of Campina Grande, Paraíba, were analyzed. Microbiological analyzes followed the protocol of current legislation for this product, where they were evaluated for the presence of total coliforms, Staphylococcus aureus, Salmonella spp. and Listeria monocytogenes. Most of the samples analyzed were above what is allowed by the regulations for total coliforms, Salmonela spp. and Listeria monocytogenes. From the results of the microbiological analyses, inadequacies of the microbiological standards of the coalho cheese sold at the central fair were demonstrated, as it presents a large amount of pathogenic microorganisms above the limit required by Brazilian legislation, which raises a public health alert.
\end{abstract}

Keywords: Dairy products; Good handling practices; Quality control.

\title{
Resumen
}

Producto típico del nordeste brasileño, el queso tipo cuajada tiene una gran importancia económica y cultural para esta región. En el estado de Paraíba, representa un importante medio de generación de ingresos para los productores de leche, la mayoría de los cuales producen quesos como la cuajada de forma artesanal. Por ello, la leche cruda se utiliza como principal materia prima para la elaboración de este manjar, tan utilizado en la gastronomía nororiental. La falta de uso de métodos de pasteurización de la matriz alimentaria se convierte en un medio favorable para la diseminación de la contaminación del producto. La ausencia de Buenas Prácticas de Manufactura, asociada con transporte, empaque y mercadeo inadecuados, deja a los consumidores expuestos al riesgo de enfermedades transmitidas por alimentos. Debido a la importancia económica y cultural en la comercialización de este producto en la ciudad evaluada, este estudio tuvo como objetivo analizar la calidad microbiológica del queso cuajo artesanal comercializado en la feria. Así, se analizaron 10 muestras de diferentes puntos comerciales de la feria del queso de la ciudad de Campina Grande, Paraíba. Los análisis microbiológicos siguieron el protocolo de la legislación vigente para este producto, donde se evaluó la presencia de coliformes totales, Staphylococcus aureus, Salmonella spp. y Listeria monocytogenes. La mayoría de las muestras analizadas estuvieron por encima de lo permitido por la normativa para coliformes totales, Salmonela spp. y Listeria monocytogenes. A partir de los resultados de los análisis microbiológicos, se demostraron insuficiencias de los estándares microbiológicos del requesón vendido en la feria central, ya que presenta una gran cantidad de microorganismos patógenos por encima del límite exigido por la legislación brasileña, lo que genera una alerta de salud pública.

Palabras clave: Productos lácteos; Buenas prácticas de manejo; Control de calidad.

\section{Introdução}

Produto tipicamente nordestino, e com mais de um século e meio de história, o queijo tipo coalho é assim chamado devido ao antigo processo de fermentação do leite cru, onde se utilizava pedaços de estômago de ruminantes para formação do coalho (Cavalcante et al., 2007; Freitas, 2011). Tido nacionalmente como iguaria, e tem como maiores produtores os estados do Ceará, Paraíba, Rio Grande do Norte e Pernambuco, alavancando a economia local e tornando-se fonte de renda de várias famílias produtoras de leite desses estados (Nassu et al, 2006).

Devido a intensa atividade pecuarista, sua elevada produção de leite e o grande número de queijarias, a região do sertão da paraíba é o maior produtor de queijo coalho do estado, embora o produto seja bastante difundido na região, não existem dados estatísticos oficiais sobre sua produção, sabe-se que essas pequenas queijarias produzem seus produtos em sua maioria de forma artesanal, com leite crú. (Medeiros, 2016; Araújo, 2017).

É denominado como queijo de coalho aquele oriundo do talho do leite cru ou pasteurizado por meio do coalho ou outros fermentos coagulantes adequados, podendo ser associada ou não pela atuação de bactérias lácteas selecionadas, podendo ser vendidos no prazo de até dez dias após sua preparação. Tem como suas principais propriedades sensoriais a consistência semidura e elástica, textura compacta e macia, coloração branco amarelado uniforme, sabor brando, discreta acidez; podendo ser salgado, 
odor ligeiramente ácido, lembrando massa coagulada, apresentando algumas olhaduras mecânicas, forma e peso variáveis, sendo gordo ou semi-gordo, dependendo do percentual de lipídios apresentados em sua composição (Sousa et al., 2014; Brasil, 2001).

Este tipo de queijo é fabricado de forma artesanal, comercializado em alguns locais do nordeste apresentam índices elevados de contaminação por bactérias patogênicas como o Staphylococcus e coliformes, além da presença de Salmonella sp., representando um perigo à saúde dos consumidores (Sousa et al., 2014: Sousa et al., 2020).

Apesar de o Brasil dispor de regras bem claras sobre a fabricação do queijo de coalho, estabelecendo a produção a partir de leite pasteurizado ou com tratamento térmico adequado, muitos produtores ainda confeccionam seus produtos com leite de vaca cru, viabilizando a contaminação por microrganismos possivelmente patogênicos (Brasil, 2001).

Estudos anteriores indicam que o consumo de queijo coalho artesanal produzidos na região Nordeste está ligado ao potencial risco de intoxicação alimentar, devido ao processamento, manipulação e comercialização inadequados desses produtos (Andrade et al., 2006; Santana et al., 2008; Alves et al., 2009; Freitas; Travassos \& Maciel, 2013; Sousa et al., 2020). Pode-se afirmar, portanto, com base nestes conhecimentos, que a qualidade do queijo Coalho artesanal produzido na Região Nordeste, é objeto de grande preocupação das autoridades sanitárias desses locais, pois tais produtos representam sério perigo à saúde de quem os consome (Cavalcante, 2005; Barros et al., 2019; Sousa et al., 2020).

Esse estudo visa alertar a população campinense sobre as condições higiênico-sanitárias dos queijos tipo coalho comercializados na feira livre de Campina Grande, PB. Para tanto, foi utilizado o regulamento técnico sobre as categorias microbiológicas para alimentos como padrão, com o intuito de avaliar a qualidade microbiológica de queijos coalhos vendidos em feiras livres da cidade de Campina Grande, PB.

\section{Metodologia}

O queijo de coalho foi adquirido de forma aleatória na feira livre de Campina Grande, no agreste paraibano, foram compradas uma amostra de cada feirante, totalizando 10 amostras, de vendedores diferentes a partir da retirada de uma fração de 100 gramas da peça inteira, utilizando a faca do próprio comerciante. Depois foram acondicionadas em sacos estéreis e transportadas em caixa isotérmica com gelo e com suas respectivas identificações (ex.: Q1, Q2, Q3, Q4, Q5, Q6, Q7, Q8, Q9, Q10). As amostras foram analisadas sequencialmente uma a uma no mesmo dia em que foram adquiridas, sendo mantidas sob refrigeração de $2^{\circ} \mathrm{C}$ até o momento de sua análise.

Os ensaios microbiológicos foram efetivados nos laboratórios de Microbiologia e Imunologia, e preparação da amostra ocorreu no Laboratório de Técnica Dietética, todos no Centro Universitário da UniFacisa.

As análises microbiológicas seguiram os protocolos sugeridos pela Resolução de Diretoria Colegiada (RDC) 12, de 02 de janeiro de 2001 e da instrução normativa 62 , de 26 de agosto de 2003. Que recomenda as análises de coliforme/g $\left(45^{\circ} \mathrm{C}\right)$, Estafilococos Coag. Pos/g, Salmonela spp./25g e Listeria monocytogenes/25g, para queijo com alta umidade e com bactérias lácticas em forma variável e abundantes (Brasil, 2003).

O método de análise utilizado para contagem de coliformes foi o proposto por Silva et al. (2017) para contagem de coliformes totais em água e alimentos. Para tanto, foi aplicada a técnica dos tubos múltiplos com preparação de diluições até a $10^{-3}$. A amostra passou por diluição seriada e homogeneização por agitação em água peptonada e inoculação em Caldo Lactosado Bile Verde Brilhante (CLBVB), que permite a fermentação do grupo de coliformes. Após a inoculação, os tubos em triplicata foram levados para estufa à $45^{\circ} \mathrm{C}\left( \pm 2^{\circ} \mathrm{C}\right)$ por $48 \mathrm{~h}( \pm 1)$. Sendo a positividade dada pela presença do gás preso no tubo de Durham.

A contagem de Listeria foi realizada segundo o proposto pelo método de plaqueamento ISO 11290-2:1998 (2004) recomendado por Silva et al. (2017). Sendo uma parte da amostra diluída em água peptonada e feita a reparação das células injuriadas em banho-maria a $20^{\circ} \mathrm{C} \pm 2^{\circ} \mathrm{C}$ por $1 \mathrm{~h} \pm 5 \mathrm{~min}$. Após este tempo uma alíquota de $0,1 \mathrm{ml}$ foi transferida para uma placa 
de Petri com Agar Listeria PALCAM, com plaqueamento em superfície. A análise foi realizada em duplicata e incubada a $35^{\circ} \mathrm{C}$ $\left( \pm 2^{\circ} \mathrm{C}\right)$ por $48 \mathrm{~h}( \pm 1)$. A positividade se deu pela formação de colônias cinzentas esverdeadas circundadas por halos castanhosescuros a preto no meio.

Para detectar Staphylococcus foi seguida a metodologia descrita por Resta \& Oliveira (2013). Para tanto, uma alíquota de 2,5g de cada amostra do alimento foram pesadas assepticamente e homogeneizadas com 22,5 $\mathrm{ml}$ de água peptonada $0,1 \%$. Depois de um período de 30 a 60 minutos sob refrigeração de $2{ }^{\circ} \mathrm{C}$, as amostras foram submetidas à homogeneização. Diluições decimais, a partir da diluição $10^{-1}$, foram preparadas em tubos contendo $9 \mathrm{ml}$ de água peptonada $0,1 \%$ estéril, até a diluição de $10^{-}$ 3. Alíquotas de 0,1 $\mathrm{ml}$ das diluições foram semeadas em ágar Vogel e Johnson em duplicata. As placas foram incubadas a 35-37 ${ }^{\circ} \mathrm{C}$ e, após $48 \mathrm{~h}$, realizadas a identificação e a contagem das colônias que apresentavam as características típicas de Staphylococcus spp. Em meio de cultivo Agar Volgel-Johnson apresenta-se negras, pequenas e com halo amarelo.

Para Salmonella spp. foi usado o Método da AOAC (2019) sugerido por Sousa et al. (2020) para presença e ausência de Salmonella em alimentos. Para tanto, a amostra foi diluída em água peptonada para pré-enriquecimento; e deixada em repouso a $35^{\circ} \mathrm{C}\left( \pm 1^{\circ} \mathrm{C}\right)$ por $18 \mathrm{~h}( \pm 1)$. Depois foi transferido $1 \mathrm{ml}$ do conteúdo para o caldo de Tetrionato e incubada à $37^{\circ} \mathrm{C}\left( \pm 1^{\circ} \mathrm{C}\right)$ por

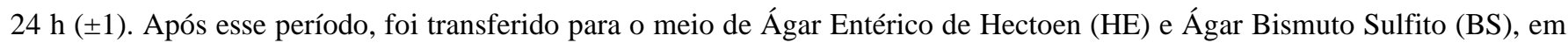
alíquotas de $0,1 \mathrm{ml}$ e novamente incubada a $37^{\circ} \mathrm{C}\left( \pm 1^{\circ} \mathrm{C}\right)$ por $24 \mathrm{~h}( \pm 1)$, esta análise foi feita por esgotamento, em duplicata. As colônias típicas no Ágar HE eram transparentes, verde-azuladas, com ou sem centro preto, e as apresentadas no Ágar BS eram colônias castanhas, cinza ou pretas, com ou sem brilho metálico.

\section{Resultados e discussão}

Os resultados encontrados nas análises são demostrados na tabela 1. Dos queijos analisados só a amostra Q1 para análise de coliformes, Staphylococcus aureus, Salmonella spp. e Listeria monocytogenes apresentou valores dentro do preconizado pela RDC $\mathrm{n}^{\circ} 12$, de 02 de janeiro de 2001, estando esse apto para o consumo humano (Anvisa, 2001). As demais amostras apresentaram valores de coliformes acima da legislação, e algumas amostras apresentaram a presença de Salmonella sp. e/ou a presença de Listeria monocytogenes, estando esses inaptos para o consumo humano, uma vez que a presença desses patógenos é prejudicial à saúde de quem os consome. 
Tabela 1 - Análise da qualidade do queijo coalho vendidos em uma feira livre do município de Campina Grande.

\begin{tabular}{|c|c|c|c|c|}
\hline \multirow[b]{2}{*}{ Amostras } & \multicolumn{4}{|c|}{ Análises } \\
\hline & $\begin{array}{c}\text { Coliformes totais à } 45^{\circ} \mathrm{C} \\
(\mathrm{NMP} / \mathrm{g})\end{array}$ & $\begin{array}{l}\text { Staphylococcus } \\
\text { aureus (UFC/g) }\end{array}$ & $\begin{array}{c}\text { Salmonella } \\
\text { spp./25g }\end{array}$ & $\begin{array}{c}\text { Listeria } \\
\text { monocytogenes } / 25 \mathrm{~g}\end{array}$ \\
\hline Q1 & $<3,0$ & Ausente & Ausente & Ausente \\
\hline Q2 & $>1,1 \times 10^{3}$ & Ausente & Presente & Presente \\
\hline Q3 & $>1,1 \times 10^{3}$ & Ausente & Presente & Ausente \\
\hline Q4 & $>1,1 \times 10^{3}$ & Ausente & Ausente & Presente \\
\hline Q5 & $>1,1 \times 10^{3}$ & Ausente & Ausente & Presente \\
\hline Q6 & $>1,1 \times 10^{3}$ & Ausente & Ausente & Ausente \\
\hline Q7 & $>1,1 \times 10^{3}$ & Ausente & Presente & Presente \\
\hline Q8 & $>1,1 \times 10^{3}$ & Ausente & Presente & Ausente \\
\hline Q9 & $>1,1 \times 10^{3}$ & Ausente & Presente & Presente \\
\hline Q10 & $>1,1 \times 10^{3}$ & Ausente & Presente & Presente \\
\hline Legislação* & $5 \times 10^{2}$ & $10^{3}$ & Ausente & Ausente \\
\hline
\end{tabular}

Q1: amostra 1; Q2: amostra 2; Q3: amostra 3; Q4: amostra 4; Q5: amostra 5; Q6: amostra 6; Q7: amostra 7; Q8:amostra 8; Q9: amostra 9; Q10: amostra 10. *Segundo a Resolução Normativa $\mathrm{n}^{\circ} 12 / 01$ que aprova regulamento técnico sobre padrões microbiológicos para alimentos.

Fonte: Dados da pesquisa (2021).

No que se refere as análises de coliformes, em $90 \%$ (n=9) das amostras constatou-se a presença desses patógenos em valores de contagem acima do limite aceitável pela legislação. Sousa et al. (2020), em sua pesquisa, avaliaram a presença destes microrganismos que indicam as condições sanitárias em queijo de coalho vendidos no município de Soledade, Paraíba. Para tanto, detectaram coliformes em valores acima do aceitável em 85,71\% das amostras. Já Santos et al. (2020) em suas avaliações microbiológicas do queijo coalho na cidade de Garanhuns, Pernambuco, identificaram a presença de coliformes termotolerante em todas as amostras dentro do permitido pela legislação quanto a seus parâmetros.

É importante frisar que os coliformes termotolerantes pertencem a um grupo de microrganismos que habita o trato intestinal do homem e de outros animais de sangue quente, portanto sua presença em alimentos indica que houve contato direto ou indireto do produto com fezes. Essa contaminação pode ocorrer pelo transporte, armazenamento e manipulação inadequados ou durante o beneficiamento (Brasil, 1996; Duarte et al., 2005; Salotti et al., 2006).

Para as análises de Staphylococcus aureus foi encontrado que em 100\% (n=10) das amostras de queijos vendidas não havia a presença desse microrganismo. O que vai de acordo com os resultados encontrados nos estados de sergipe e paraíba respectivamente por Alexandre et al. (2020) e Sousa et al. (2020), onde todas as amostras de queijos deram negativo para Staphylococcus aureus.

Em discrepância com os nossos resultados, Santos (2019) detectou cepas de S. aureus em 100\% das amostras deste mesmo alimento, com contagens variando de $1,09 \times 10^{\natural}$ a $1,3 \times 10^{8} \mathrm{UFC} / \mathrm{g}$. A legislação vigente para este produto não permite valores com contagens acima de $10^{3}$ (Anvisa, 2001).

Segundo Montanari et al. (2015) infecções por Staphylococcus coagulase positiva são causadoras de desordens intestinais. No caso específico do leite, os tratamentos térmicos disponíveis não são capazes de inativar estas enterotoxinas, constituindo risco potencial para o consumidor (Lamaita et al., 2005).

Para determinação de Salmonella spp, encontramos que $60 \%(\mathrm{n}=6)$ das amostras apresentaram cepas características 
para este patógeno. Feitosa et al. (2003) também observaram este microrganismo em quantidade inferior $(9,1 \%)$ em queijos produzido no estado potiguar. Em contrapartida, Camargo et al. (2020) em seus estudos não observaram esta bactéria em cinco amostras de suas amostras analisadas de queijo-de-minas vendidos em Fernandópolis (SP).

Por ser capaz de induzir processos infecciosos por via alimentar, a presença desse microrganismo no queijo classificao como produto inapropriado para ingesta pois, a pasteurização é capaz de eliminá-la, a falta desse processo evidencia a prevalência dessa bactéria em queijos coalho tipo artesanal. A legislação vigente padroniza a ausência de Salmonella spp. em alimentos para comercialização e ingesta, uma vez que a mesma sobrevive a acidez estomacal, chegando ao intestino do hospedeiro onde inicia a infecção. Os sintomas mais comuns são febre baixa, dor estomacal, vômito e diarreia, na maioria das vezes não requer hospitalização. Mas se os sintomas perdurarem por mais de 72 horas o médico deve ser consultado, pois o hospedeiro pode ter contraído a forma mais virulenta da bactéria, como a Salmonella Typhi, que pode durar até oito semanas causar sepse e levar a morte (Brasil, 2001; Alves, 2012; Sousa et al. 2014).

$\mathrm{Na}$ análise para Listeria monocytogenes houve crescimento de colônias características em $60 \%$ (n=6) das amostras de queijos vendidos. Segundo Pinto et al. (2011), em suas pesquisas obtiveram resultados aceitáveis quando observou ausência desse patógeno em queijos vendidos no município de Santa Helena (PR).

Silva et al. (2011) confirmam que a ocorrência desse microrganismo em leites e derivados não é constante, pois a doença causada é preocupante. Os principais sintomas de infecção por Listeria monocytogenes são: febre, perda de apetite problemas gastrointestinais, como náuseas, vômitos e diarreia; e em casos mais graves e raros, podendo se espalhar pela corrente sanguínea e chegar no sistema nervoso, onde pode provocar meningite, que é uma inflamação nas membranas que envolvem o cérebro (Fda, 2009).

Dessa forma deve-se evitar a contaminação durante o processo de fabricação desses alimentos. Alguns cuidados como: cozinhar bem alimentos de origem animal, lavar vegetais crus em água corrente, não misturar alimentos cozinhados com alimentos crus; não tomar leite cru, lavar bem as mãos, utensílios e bancadas, antes e após o manuseio podem reduzir este tipo de contaminação (Gonçalves et al., 2017).

\section{Conclusão}

A partir das análises microbiológicas foi possível concluir que $90 \%$ das amostras de queijo coalho adquiridas na feira livre de Campina Grande-PB, se mostraram impróprias para o consumo da população que mora nessa cidade e consome esse alimento diariamente. A má qualidade microbiológica do queijo contaminado por Coliformes, Staphylococcus coagulase positiva, Salmonella spp. e Listeria monocytogenes, é um risco para a saúde pública, uma vez que tais microrganismos são reconhecidos como causadores de danos à saúde do consumidor, tendo como principais sintomas dores abdominais, dor de cabeça, febre, infecção de pele e nos casos mais graves podendo levar a morte. É importante que mais pesquisas sejam realizadas e que englobe um número maior de amostras com mais comerciantes de queijo da feira central deste município e medidas sejam adotadas pelas entidades públicas para a adequação normativa desses feirantes.

Sendo assim, apropriando-se dos resultados desta pesquisa, torna-se pertinente um trabalho de orientação, treinamento e adequação de normas junto aos feirantes e produtores de queijo, a fim de evitar surtos de doenças transmitidas por alimentos (DTA's) nesta cidade. Servindo de alerta para a população em relação aos perigos do consumo de alimentos fabricados artesanalmente e comercializado de maneira informal sem refrigeração, exposição e manipulação inadequados.

Nota-se também, a necessidade de uma maior atenção das autoridades sanitárias municipais, orientando os comerciantes para que se adequem as boas práticas de manipulação de acordo com a legislação vigente, desde a fabricação até a comercialização do produto, uma vez que durante a aquisição dos produtos para tal pesquisa, foi visualizado o total abandono 
dos feirantes pela administração pública desta cidade. Uma vez que tais feirantes não dispõem de um local com higiene, arquitetura e equipamentos adequadas, tais como: expositores refrigerados e freezers. O que poderia evitar a contaminação por fatores extrínsecos como: temperatura ambiente, exposição inadequada, poeira, saliva, suor e o próprio local de exposição, são grande agentes de contaminação.

\section{Referências}

Alexandre, A. P. S., Camatari, F. O. S., Santana, L. C. L. A., \& Silva, M. A. A. P. (2020). Efeito da acidificação direta e microbiana sobre as propriedades químicas, físicas e microbiológicas do queijo de manteiga. Research, Society and Development, 9(8), e650985997.

Alves, A. R. F. (2012). Doenças alimentares de origem bacteriana. 87f. Dissertação (Mestrado em Ciências Farmacêuticas). Faculdade de Ciências da Saúde, Universidade Fernando Pessoa, Porto.

Alves, M.C.A. et al. (2009). Qualidade microbiológica do leite cru e de queijo de Coalho comercializados informalmente na cidade de São Luís - MA. Pesquisa em Foco, 17(12).

Andrade, A. A. (2006). Estudo do perfil sensorial, físico-químico e aceitação do queijo de coalho produzido no estado do Ceará. 2006.127 f. Dissertação (Mestrado em tecnologia de alimentos) - Universidade Federal do Ceará, Fortaleza.

Anvisa. Agência Nacional de Vigilância Sanitária. Resolução-RDC No 12, de 02 de janeiro de 2001 - Regulamento Técnico Sobre Padrões Microbiológicos Para Alimentos. 2001.

AOAC - Association of Official Agricultural Chemists. (2019). Official methods of analysis of Association of Official Agricultural Chemists. (21th ed.), Ass. Off. Analytical. Chem., Washington, USA.

Araújo, L. M. (2017) Avaliação de propriedades probióticas de bactérias ácido láticas isoladas de queijo de coalho do sertão da paraíba. 2017.72 f. Dissertação (mestrado em ciências biológicas) - Universidade Federal de Pernambuco, Pernambuco.

Barros, D, Machado, E., Moura, D., Fonte, R., Ferreira, S., \& Bezerra, R. (2019). Aspectos do queijo de coalho com ênfase na importância das Boas Práticas de Fabricação no sistema de produção. Brazilian Journal of Development, 5, 67-93.

Brasil. Instrução normativa ${ }^{\circ}$ 30, de 26 de junho de 2001. Regulamento Técnico de Identidade e Qualidade de Manteiga da Terra ou Manteiga de Garrafa, Queijo de Coalho e Queijo de Manteiga. Diário Oficial da União, 13, 16.

Brasil. Ministério da Agricultura. Portaria n 146, de 7 de março de 1996. Regulamento Técnico de Identidade e Qualidade de Queijos. Diário Oficial da República Federativa do Brasil, Brasília, DF, 11 mar. 1996, Seção 1, 3977-3978.

Camargo, A. et al., (2020). Qualidade microbiológica do queijo tipo minas “frescal” comercializado na cidade de Fernandópolis-SP. Brazilian Journal of Health Review, 3, 10370-10382.

Cavalcante, J. F. M. (2005). Sistema de apoio à decisão na produção de leite e queijo coalho com segurança alimentar. Tese (Doutorado em ciência e tecnologia dos alimentos) - Universidade Federal de Viçosa, 16.

Cavalcante, J. F. M., et al. (2007). Processamento do queijo coalho regional empregando leite pasteurizado e cultura lática endógena Ciênc. Tecnologia dos Alimentos, 27(1): 205-214.

Duarte, D. A. M. , Schuch, D. M. T. , Santos, S. B. , Ribeiro, A. R., Vasconcelos, A. M. M. , Silva, J. V. D. \& Mota, R. A. (2005). Pesquisa de Listeria monocytogenes e microrganismos indicadores higiênicos-sanititários em queijo de coalho produzido e comercializado no Estado de Pernambuco. Arquivos do Instituto Biológico, 72, .297-302.

Feitosa, t., Borges, M. F. , Nassu, R. T., Azevedo, E. H. F. \& Muniz, C. R. (2003). Pesquisa de Salmonella sp., Listeria sp. e microrganismos indicadores higiênicos-sanitários em queijos produzidos no Estado do Rio Grande do Norte. Ciência e Tecnologia de Alimentos, 23, 162-165. Suplemento 1.

Freitas, W. C. de. (2011). Aspectos higiênicos-sanitários, físico-químicos e microbiota lática, de leite cru, queijo de coalho e soro de leite produzidos no estado da paraíba. 18, 26p. tese (doutorado em ciências e tecnologia de alimentos). Universidade Federal da Paraíba, UFPB, Paraíba.

Freitas, W.C., Travassos, A. E. R.\& Maciel, J. F. (2013). Avaliação microbiológica e físico- química de leite cru e queijo de coalho produzidos no estado da Paraíba. Revista Brasileira de Produtos Agroindustriais, 15.

Fda. Food And Drug Administration Of U.S. 〈http://www.fda.gov/default.htm>.

Gonçalves, M., Furtado, R., Coelho, A., Correia, B. C \& Valente, A. (2017) Presença de listeriamonocytogenes em queijos de pasta mole da região a sul do Tajo. Journal Public Health, 35, 43.

Lamaita, H. C. , Cerqueira, M. M. O. P. , Carmo, L. S. , Santos, D. A. , Penna, C. F. A. M. \& Souza, M. R. (2005). Contagem de Staphylococcus sp. e detecção de enterotoxinas estafilocócicas e toxina da síndrome do choque tóxico em amostra de leite cru refrigerado. Arquivo Brasileiro de Medicina Veterinária e Zootecnia, 57, 702-709.

Medeiros, R. S. (2016). Parâmetros de qualidade do Queijo de Coalho produzido na Paraíba: indicadores químicos e microbiológicos. Tese (Doutorado). Faculdade de Ciências e Tecnologia Universidade Nova de Lisboa. 
Ministério da Agricultura. Instrução Normativa No 62, de 26 de agosto de 2003. Métodos Analíticos Oficiais para Análises Microbiológicas para Controle de Produtos de Origem Animal e Água. Diário Oficial da União de 18/09/2003.

Montanari, A.S., Romão, N.F., Sobral, F.O.S., Marmitt, B.G., Silva, F.P.S. \& Correio, T.C.A.M. (2015). Avaliação da qualidade microbiológica de sashimis de salmão, preparados e comercializados em restaurantes japoneses no município de Ji-Paraná - RO. South American Journal of Basic Education, Technical and Technological, 2(1): 4-16.

Nassu, R. T., Andrade, A. S. A. de, Silva, A. C. et al. (2006). Caracterização físico-química de queijos regionais produzidos no estado do Rio Grande do Norte. Revista do instituto de laticínios cândido tostes. 61, 3030-305.

Pinto, S. G. F, Souza, M, Saling, S. \& Moura, C. A. (2011). Qualidade microbiológica de queijo minas frescal comercializado no município de Santa Helena, PR., Brasil. Arquivo do Instituto de Biologia, 78, 191-198.

Resta, M. S. A. \& Oliveira, T. C. R. M. D. (2013). Avaliação do padrão estafilococos coagulase positiva estabelecido pela legislação brasileira para massas alimentícias. Brazilian Journal of Food Technology, 16(4), 319-325.

Salotti, B. M. , Carvalho, A. C. F. B. , Amaral, L. A. , Vidal-Martins, A. M. C. \& Cortez, A. L. (2006). Qualidade microbiológica do queijo minas frescal comercializado no Município de Jaboticabal, SP, Brasil. Arquivos do Instituto de Biológico, 73, 171-175.

Santana, R. F. et al. (2008). Qualidade microbiológica de queijo-coalho comercializado em Aracaju, SE. Arquivo Brasileiro de Medicina veterinária e Zootecnia., $60,1517-1522$.

Santos, J. G. (2019). Análise microbiológica do queijo coalho produzido por usuários da Fazenda da Esperança em Lagarto - Sergipe. 2019. Trabalho de conclusão de curso (graduação em farmácia) - Universidade Federal de Sergipe, Aracajú.

Santos, N. et al. (2020). Perfil instrumental de textura e avaliação microbiológica de queijo coalho comercializado em feira livre. Research, Society and Development, $9(5)$.

Silva, S. A, Aragon, C. C, Santana, W. H. E, Destro, T. M, COSTA, R. M. \& Alegro, A. C. L. (2011). Listeria monovytogenes em leite e produtos lácteos no Brasil: uma revisão. UNOPAR. Científica Ciências Biológicas e da Saúde, Paraná.

Silva, N., Junqueira, A. C. V., Silveira, A. F. N., Taniwaki, H. M, Gomes, R. A.R. \& Okazaki, M. M. (2017). Manual de métodos de análises microbiológica de alimentos e água.(5a ed.), Blucher.

Sousa, A. Z. B, Abrantes, M. R, Sakamoto, S. M. , Silva, J. B. A, Lima, P. O, Lima, R. N. , Rocha, M. O. C . \& Passos, Y. D. B. (2014). Aspectos físicoquímicos e microbiológicos do queijo tipo coalho comercializado em estados do nordeste do Brasil. Arquivo Brasileiro Instituto de Biologia. 81,.30-35.

Sousa, L., Cavalcanti, M., Sousa, M. \& Paz, E. (2020). Avaliação microbiológica do queijo artesanal produzido e comercializado em uma cidade do interior da Paraíba. Research, Society and Development, 9(8). 